\title{
Propelinear 1-perfect codes from quadratic functions
}

\author{
Denis S. Krotov and Vladimir N. Potapov
}

\begin{abstract}
Perfect codes obtained by the Vasil'ev-Schönheim construction from a linear base code and quadratic switching functions are transitive and, moreover, propelinear. This gives at least $\exp \left(c N^{2}\right)$ propelinear 1-perfect codes of length $N$ over an arbitrary finite field, while an upper bound on the number of transitive codes is $\exp \left(C(N \ln N)^{2}\right)$.
\end{abstract}

Index Terms-perfect code, propelinear code, transitive code, automorphism group.

\section{INTRODUCTION}

$\mathbf{U}$ SUALLY, a group code is defined as a subgroup of the additive group of a finite vector space. There are alternative approaches [12], [16], [13], [6], [5], [7], [9] that allow to relate the codewords of a code with the elements of some group. Usually, the mapping from the group to the code is required to satisfy some metric properties, because the distance is what is very important for error-correcting codes. One of the approaches considers so-called propelinear codes, introduced in [16] for the binary space. The codewords of a propelinear code $C$ are in one-to-one correspondence with a group $G$ of isometries of the space that acts regularly on the code itself. In other words, given some fixed codeword $v \in C$ (say, the all-zero word), every other codeword can be uniquely written as $g(v), g \in G$. Every propelinear code is transitive; that is, it is an orbit of a group of isometries of the space (for a transitive code in general, this group is not required to act regularly).

In the current paper, we will prove that the number of nonequivalent propelinear codes with the same parameters, namely, the parameters of 1-perfect codes over an arbitrary finite field, grows at least exponentially with respect to the square of the code length (Corollary 1). By the order of the logarithm, this number is comparable with the total number of propelinear codes (Theorem 2). In contrast, there is only one (up to equivalence) linear 1-perfect code for each admissible length, but the number of non-linear 1-perfect codes grows doubly-exponentially [19], [17].

For the case $q=2$, an exponential lower bound (with respect to the square root of the code length, to be more accurate) on the number of transitive and the number of

D. Krotov and V. Potapov are with the Sobolev Institute of Mathematics, pr. Akademika Koptyuga 4, Novosibirsk 630090, Russia, and with the Novosibirsk State University, Pirogova 2, Novosibirsk 630090, Russia; e-mail: krotov@math.nsc.ru, vpotapov@math.nsc.ru.

Manuscript received October 19, 2013; revised January 22, 2014. The work was supported in part by the RFBR (project 13-01-00463), by the Ministry of education and science of Russian Federation (project 8227), and by the Target program of SB RAS for 2012-2014 (integration project No. 14). The material in this paper was presented in part at the Mal'tsev Meeting, Novosibirsk, Russia, November 12-16, 2012.

Copyright (c) 2014 IEEE. Personal use of this material is permitted. However, permission to use this material for any other purposes must be obtained from the IEEE by sending a request to pubs-permissions@ieee.org

Digital Object Identifier 10.1109/TIT.2014.2303158 propelinear 1-perfect codes was firstly established in [15] and [2], respectively. Here, we will show how to improve the lower bound and generalize it to an arbitrary prime power $q$, using a rather simple construction. Some other constructions of transitive and propelinear perfect codes can be found in [3], [8], [18], [1].

Section III contains definitions and auxiliary lemmas. In Section III we formulate the main results of the paper. The main theorem is proven in Section IV. In Section V, we consider some remarks and examples concerning the structure of the group related to a propelinear code, survey the transitive (propelinear) Vasil'ev codes of length 15, and discuss a problem about functions that can result in transitive codes.

\section{PRELIMINARIES}

Let $F$ be a finite field of order $q$, where $q$ is a power of prime; let $F^{n}$ be the vector space of all words of length $n$ over the alphabet $F$. An arbitrary subset of $F^{n}$ is referred to as a code. A code is linear if it is a vector subspace of $F^{n}$. A code $C \subset F^{n}$ is called 1-perfect if for every word $v$ from $F^{n}$ there is exactly one $c$ in $C$ agreeing with $v$ in at least $n-1$ positions. It is well known that 1-perfect codes exist if and only if $n=\left(q^{k}-1\right) /(q-1)$ for some integer $k$, see e.g. [10].

\section{A. Vasil'ev-Schönheim construction}

Let $H \subset F^{n}$, and let $f: H \rightarrow F$ be an arbitrary function. Define the set

$$
\begin{array}{r}
C(H, f)=\left\{\left(\left(v_{\alpha}\right)_{\alpha \in F}, z\right): v_{\alpha} \in F^{n}, \sum_{\alpha \in F} v_{\alpha}=c \in H,\right. \\
\left.z=\sum_{\alpha \in F} \alpha\left|v_{\alpha}\right|+f(c)\right\}
\end{array}
$$

where $\left(v_{\alpha}\right)_{\alpha \in F}$ is treated as the concatenation of the words $v_{\alpha}$ (which will be referred to as blocks) in some prefixed order, $\left|v_{\alpha}\right|$ is the sum of all $n$ elements of $v_{\alpha}$. If $H$ is a 1-perfect code, then $C(H, f)$ is a 1-perfect code in $F^{q n+1}$, known as a Schönheim code [17] (in the case $q=2$, as a Vasil'ev code [19]). Clearly, the set $C(H, f)$ essentially depends on the choice of the function $f$ :

Lemma 1: For a fixed $H$, different functions $f$ result in different $C(H, f)$.

Proof: The graph of the function $f$ can be reconstructed from the set $C(H, f)$ :

$$
\begin{array}{r}
\{(x, f(x)): x \in H\}=\left\{\left(\sum_{\alpha \in F} v_{\alpha}, z-\sum_{\alpha \in F} \alpha\left|v_{\alpha}\right|\right):\right. \\
\left.\left(\left(v_{\alpha}\right)_{\alpha \in F}, z\right) \in C(H, f)\right\} .
\end{array}
$$

Hence, $C(H, f)=C\left(H, f^{\prime}\right)$ implies $f=f^{\prime}$. 


\section{B. Automorphisms, equivalence, transitivity, propelinearity}

The Hamming graph $G\left(F^{n}\right)$ is defined on the vertex set $F^{n}$; two words are connected by an edge if and only if they differ in exactly one position. It is known (see, e.g., [4, Theorem 9.2.1]) that every automorphism $\Pi$ of $G\left(F^{n}\right)$ is composed from a coordinate permutation $\pi$ and alphabet permutations $\psi_{i}$ in each coordinate: $\Pi(x)=\left(\psi_{1}\left(x_{\pi^{-1}(1)}\right), \ldots, \psi_{n}\left(x_{\pi^{-1}(n)}\right)\right)$. Two codes are said to be equivalent if there is an automorphism of $G\left(F^{n}\right)$ that maps one of the codes into the other. Note that the algebraic properties of the code, such as being a linear or affine subspace, are not invariant with respect to this combinatorial equivalence, in general. The automorphism group $\operatorname{Aut}(C)$ of a code $C$ consists of all automorphisms of $G\left(F^{n}\right)$ that stabilize (fix set-wise) $C$. A code $C$ containing the all-zero word $\overline{0}$ is transitive if for every codeword $a$ there exists $\varphi_{a} \in \operatorname{Aut}(C)$ that sends $\overline{0}$ to $a$. If, additionally, the set $\left\{\varphi_{a}: a \in C\right\}$ is closed under composition (that is, for all $a$ and $b$ from $C$ we have $\varphi_{a} \varphi_{b}=\varphi_{c}$, where $\left.c=\varphi_{a} \varphi_{b}(\overline{0})\right)$, then $C$ is a propelinear code, see e.g. [2].

\section{Quadratic functions}

Assume $H$ is a subspace of $F^{n}$. A function $f: H \rightarrow F$ is called quadratic if it can be represented as a polynomial of degree at most 2 .

We will use the following elementary property of the quadratic functions (actually, it is a characterizing property).

Lemma 2: Let $H$ be a subspace of $F^{n}$. If $f: H \rightarrow F$ is a quadratic function, then for every $c \in H$ there exist $\beta_{0}^{c}, \beta_{1}^{c}, \ldots, \beta_{n}^{c} \in F$ such that

$$
\begin{gathered}
f(x+c)=f(x)+\beta_{0}^{c}+\beta_{1}^{c} x_{1}+\ldots+\beta_{n}^{c} x_{n} \\
\text { for all } x=\left(x_{1}, \ldots, x_{n}\right) \in H .
\end{gathered}
$$

Moreover, $\beta_{i}^{c}, i \in\{1, \ldots, n\}$, depends linearly on $c$ :

$$
\beta_{i}^{c+d}=\beta_{i}^{c}+\beta_{i}^{d}
$$

Proof: The difference of $\left(x_{i}+c_{i}\right)\left(x_{j}+c_{j}\right)$ and $x_{i} x_{j}$ has degree at most 1 . Moreover, the coefficients at $x_{i}$ and $x_{j}$ in this difference depend linearly on $c$. Hence, the same is true for the difference of $P(x+c)$ and $P(x)$ for every polynomial $P$ of degree at most 2 .

Lemma 3: Let $H$ be an $m$-dimensional subspace of $F^{n}$. There are at least $q^{m^{2} / 2}$ different quadratic functions from $H$ to $F$.

Proof: Obviously, a linear transformation of the space does not affect to the property of a function to be quadratic. Hence, we can assume without loss of generality that $H$ consists of the words of length $n$ with zeroes in the last $n-m$ positions. Then, the number of different quadratic functions is the number of polynomials of degree at most 2 in the first $m$ variables, i.e., $q^{m(m-1) / 2+m+m+1}$ for $q>2$ and $q^{m(m-1) / 2+m+1}$ for $q=2\left(\right.$ when $\left.x_{i}^{2} \equiv x_{i}(\bmod 2)\right)$.

\section{MAIN RESUlTS}

\section{A. Lower bound}

In the Section IV, we will prove the following theorem.
Theorem 1: If $H \subset F^{n}$ is a linear $q$-ary code and $f$ : $H \rightarrow F$ is a quadratic function, $f(\overline{0})=0$, then $C(H, f)$ is a propelinear code of length $N=q n+1$.

Corollary 1: The number of nonequivalent propelinear 1perfect $q$-ary codes of length $N=\left(q^{k}-1\right) /(q-1)$ obtained by the Vasil'ev-Schönheim construction is at least $q^{\frac{N^{2}}{2 q^{2}}+O(N \ln N)}$.

Proof: As follows from Theorem 1, Lemma 1, and Lemma 3, the number of different propelinear 1-perfect codes of type $C(H, f)$ is at least $q^{\frac{m^{2}}{2}}$, where $m=n-\log _{q}(n q-$ $n+1)$ and $n$ is the length of $H$. Since $N=q n+1$, we see that $q^{\frac{m^{2}}{2}}=q^{\frac{N^{2}}{2 q^{2}}+O(N \ln N)}$. To evaluate the number of nonequivalent codes, we divide this number by the number $N !(q !)^{N}=q^{O(N \ln N)}$ of all automorphisms of $F^{N}$ and find that this does not affect on the essential part of the formula.

\section{B. Upper bound}

To evaluate how far our lower bound on the number of transitive (propelinear) 1-perfect codes can be from the real value, we derive an upper bound:

Theorem 2: (a) The number of different transitive codes in $F^{N}$ does not exceed $2^{\left(N \log _{2} N\right)^{2}(1+o(1))}$. (b) The number of different propelinear codes in $F^{N}$ does not exceed $q^{N^{2} \log _{2} N(1+o(1))}$.

Proof: Since every subgroup of $\operatorname{Aut}\left(F^{N}\right)$ is generated by at most $\log _{2}\left|\operatorname{Aut}\left(F^{N}\right)\right|$ elements, the number of subgroups is less than $\left|\operatorname{Aut}\left(F^{N}\right)\right|^{\log _{2}\left|\operatorname{Aut}\left(F^{N}\right)\right|}=2^{\left(N \log _{2} N\right)^{2}(1+o(1))}$ (recall that $\left.\left|\operatorname{Aut}\left(F^{N}\right)\right|=(q !)^{N} N !=N^{N(1+o(1))}\right)$. Since every transitive code $C$ containing $\overline{0}$ is uniquely determined by its automorphism group (indeed, $C$ is the orbit of $\overline{0}$ under $\operatorname{Aut}\left(F^{N}\right)$ ), statement (a) follows.

The automorphisms assigned to the codewords of a propelinear code $C$ form a group of order $|C| \leq q^{N}$. It is generated by at most $\log _{2} q^{N}=N \log _{2} q$ elements; each of them can be chosen in less than $\left|\operatorname{Aut}\left(F^{N}\right)\right|=N^{N(1+o(1))}$ ways; (b) follows.

\section{Proof of Theorem 1}

Let $H \subset F^{n}$ be a linear code and let $f: H \rightarrow F$ be a quadratic function. The key point in the proof is the following simple statement.

Lemma 4: Let $f^{\prime}(x)=f(x)+\beta x_{j}$ for some $j \in$ $\{1, \ldots, n\}, \beta \in F$. Then $C\left(H, f^{\prime}\right)=\Pi_{j}^{\beta} C(H, f)$ where $\Pi_{j}^{\beta}$ is the coordinate permutation that sends the $j$ 'th coordinate of the block $v_{\alpha+\beta}$ to the $j$ 'th coordinate of the block $v_{\alpha}$ for all $\alpha \in F$ and fixes the other coordinates.

Proof: Let us consider the codeword $x=\left(\left(v_{\alpha}\right)_{\alpha \in F}, z\right)$ of $C(H, f)$. It satisfies $z=\sum_{\alpha \in F} \alpha\left|v_{\alpha}\right|+f(c)$. After the coordinate permutation $\Pi_{j}^{\beta}$, we obtain the word $y=\Pi_{j}^{\beta} x=$ $\left(\left(u_{\alpha}\right)_{\alpha \in F}, z\right)$ where for all $\alpha$ the word $u_{\alpha}$ coincides with $v_{\alpha}$ in all positions except the $j$ th, $u_{\alpha, j}$ which is equal to $v_{\alpha+\beta, j}$. 
Now we have

$$
\begin{aligned}
z & =\sum_{\alpha \in F} \alpha\left|v_{\alpha}\right|+f(c) \\
& =\sum_{\alpha \in F} \sum_{k \neq j} \alpha v_{\alpha, k}+\sum_{\alpha \in F} \alpha v_{\alpha, j}+f(c) \\
& =\sum_{\alpha \in F} \sum_{k \neq j} \alpha u_{\alpha, k}+\sum_{\alpha \in F} \alpha u_{\alpha-\beta, j}+f(c) \\
& =\sum_{\alpha \in F} \sum_{k \neq j} \alpha u_{\alpha, k}+\sum_{\alpha \in F}(\alpha+\beta) u_{\alpha, j}+f(c) \\
& =\sum_{\alpha \in F} \sum_{k=1}^{n} \alpha u_{\alpha, k}+\beta \sum_{\alpha \in F} u_{\alpha, j}+f(c) \\
& =\sum_{\alpha \in F} \alpha\left|u_{\alpha}\right|+f(c)+\beta c_{j},
\end{aligned}
$$

(we used that $\left.c=\left(c_{1}, \ldots, c_{n}\right)=\sum v_{\alpha}=\sum u_{\alpha}\right)$ which proves that $\Pi_{j}^{\beta}(x) \in C\left(H, f^{\prime}\right)$.

Now denoting $\Pi^{c}=\Pi_{1}^{\beta_{1}^{c}} \Pi_{2}^{\beta_{2}^{c}} \ldots \Pi_{n}^{\beta_{n}^{c}}$, where the coefficients $\beta_{j}^{c}$ are from (1), we get the following fact, which immediately proves the transitivity of the code:

Lemma 5: For every codeword $w=\left(\left(w_{\alpha}\right)_{\alpha \in F}, z\right)$ of $C(H, f)$, the transform $\Phi_{w}(v)=w+\Pi^{c}(v)$, where $c=$ $\sum_{\alpha \in F} w_{\alpha}$, is an automorphism of $C(H, f)$, which sends the all-zero word to $w$.

Proof: Consider $v=\left(\left(v_{\alpha}\right)_{\alpha \in F}, s\right)$ from $C(H, f)$. It satisfies $s=\sum_{\alpha \in F} \alpha\left|v_{\alpha}\right|+f(d)$, where $d=\sum_{\alpha} v_{\alpha}$. Applying Lemma 4 with $j=1, \ldots, n$, we see that $\Pi^{c}(v)=$ $\left(\left(u_{\alpha}\right)_{\alpha \in F}, s\right)$ satisfies $s=\sum_{\alpha \in F} \alpha\left|u_{\alpha}\right|+f(d)+\beta_{1}^{c} d_{1}+$ $\ldots+\beta_{n}^{c} d_{n}$, where $d=\left(d_{1}, \ldots, d_{n}\right)=\sum_{\alpha} u_{\alpha}$. Adding $w=\left(\left(w_{\alpha}\right)_{\alpha \in F}, z\right)$, we obtain $w+\Pi^{c}(v)=\left(\left(w_{\alpha}+u_{\alpha}\right), r\right)$, where

$$
\begin{aligned}
r= & \sum_{\alpha \in F} \alpha\left|u_{\alpha}\right|+f(d)+\beta_{1}^{c} d_{1}+\ldots+\beta_{n}^{c} d_{n} \\
& +\sum_{\alpha \in F} \alpha\left|w_{\alpha}\right|+f(c) \\
= & \sum_{\alpha \in F} \alpha\left|u_{\alpha}+w_{\alpha}\right|+f(d+c)-\beta_{0}^{c}+f(c) .
\end{aligned}
$$

But $f(c)=f(\overline{0})+\beta_{0}^{c}$, as we see from (1). Since $f(\overline{0})=0$, we have proved that $w+\Pi^{c}(v)$ belongs to $C(H, f)$.

So, we get the transitivity. It remains to prove that the set of $\Phi_{w}, w \in C(H, f)$ is closed under composition.

Lemma 6: For every $c, d \in H$ the composition $\Pi^{c} \Pi^{d}$ equals $\Pi^{c+d}$.

Proof: As follows directly from the definitions of $\Pi^{c}$ and $\Pi_{i}^{\beta}$,

$$
\begin{aligned}
\Pi^{c} \Pi^{d} & =\Pi_{1}^{\beta_{1}^{c}} \ldots \Pi_{n}^{\beta_{n}^{c}} \Pi_{1}^{\beta_{1}^{d}} \ldots \Pi_{n}^{\beta_{n}^{d}} \\
& =\Pi_{1}^{\beta_{1}^{c}} \Pi_{1}^{\beta_{1}^{d}} \Pi_{2}^{\beta_{2}^{c}} \Pi_{2}^{\beta_{2}^{d}} \ldots \Pi_{n}^{\beta_{n}^{c}} \Pi_{n}^{\beta_{n}^{d}} .
\end{aligned}
$$

By the definition of $\Pi_{i}^{\beta}$, we have $\Pi_{i}^{\beta_{i}^{c}} \Pi_{i}^{\beta_{i}^{d}}=\Pi_{i}^{\beta_{i}^{c}+\beta_{i}^{d}}$. But, by Lemma $2, \beta_{i}^{c}+\beta_{i}^{d}=\beta_{i}^{c+d}$. Finally, we have $\Pi^{c} \Pi^{d}=$ $\Pi_{1}^{\beta_{1}^{c+d}} \ldots \Pi_{n}^{\beta_{n}^{c+d}}=\Pi^{c+d}$.

Now, consider $w=\left(\left(w_{\alpha}\right)_{\alpha \in F}, z\right)$ and $v=\left(\left(v_{\alpha}\right)_{\alpha \in F}, s\right)$ form $C(H, f)$. Denote $c=\sum_{\alpha} w_{\alpha}$ and $d=\sum_{\alpha} v_{\alpha}$; observe that the permutation $\Pi^{c}$ will not change the value of the last sum. Then,

$$
\begin{aligned}
\Phi_{w} \Phi_{v}(\cdot) & =w+\Pi^{c}\left(v+\Pi^{d}(\cdot)\right) \\
& =w+\Pi^{c}(v)+\Pi^{c}\left(\Pi^{d}(\cdot)\right)=u+\Pi^{e}(\cdot),
\end{aligned}
$$

where $u=\left(\left(u_{\alpha}\right)_{\alpha \in F}, t\right)=w+\Pi^{c}(v), e=\sum_{\alpha} u_{\alpha}=c+d$. This completes the proof of the theorem.

\section{REMARKS, EXAMPLES, AND FURTHER RESEARCH}

\section{A. On the group related to $C(H, f)$}

As follows from the definition, to every codeword $v$ of a propelinear code $C$ there corresponds an automorphism $\Phi_{v}$ of $C$ and the set $\left\{\Phi_{v}: v \in C\right\}$ forms a subgroup of the automorphism group of $C$. Although such a subgroup, a propelinear structure, is not unique in general (see [1] and also Remark 2 below), in the previous section we explicitly defined a variant of the choice of $\Phi_{v}$ for every $v \in C(H, f)$. Below, we provide two remarks with examples about the propelinear structure defined in the previous section.

Remark 1: For every $v \in C(H, f)$, the element $\Phi_{v}$ has order $1, p$, or $p^{2}$, where $p$ is the prime that divides $q$. Indeed, every permutation $\Pi^{c}$ is of order 1 or $p$; hence, $\left(\Phi_{v}\right)^{p}$ corresponds to the identity permutation and has order 1 or $p$.

As an example, we consider the (non-perfect) code $C(H, f)$ constructed with the following parameters: $q=2, n=2$, $H=F^{2}, f\left(x_{1}, x_{2}\right)=x_{1} x_{2}$. From (1) we find $\beta_{1}^{01}=1$, $\beta_{2}^{01}=0, \beta_{1}^{10}=0, \beta_{2}^{10}=1, \beta_{1}^{11}=1, \beta_{2}^{11}=1$. The group of automorphisms related with the propelinear code $C(H, f)$ is generated by three elements $\Phi_{u}, \Phi_{v}, \Phi_{w}$ with $u=(11001), v=(10000), w=(10101)$ and the corresponding coordinate permutations $\Pi^{11}=(13)(24)$, $\Pi^{10}=(24), \Pi^{00}=$ Id. The first element $\Phi_{u}$ generates a cycle with the corresponding codewords (00000), (11001), (11110), (00111). The second generating element $\Phi_{v}$ adds four more codewords: (1000 0), (00 011), (01 110), (11 101); the corresponding automorphisms are of order 2 . The group generated by $\Phi_{u}$ and $\Phi_{v}$ is described by the orders of $\Phi_{u}$, $\Phi_{v}$ and the identity $\Phi_{v} \Phi_{u} \Phi_{v}=\left(\Phi_{u}\right)^{-1}$, and it is isomorphic to the dihedral group $D_{4}$. The last generating element $\Phi_{w}$ commutes with all other elements and has order 2 . It follows that the group of automorphisms related with $C(H, f)$ is isomorphic to the direct product $D_{4} \times Z_{2}$, where $Z_{2}$ is the cyclic group of order 2 .

Remark 2: If $H \neq F^{n}$, then there is more than one quadratic representations of every quadratic function on $H$. The coefficients $\beta_{i}^{c}$ and, as follows, the subgroup $\left\{\Phi_{v}\right.$ : $v \in C\}$ of the automorphism group of the code depend on the representation; so, there are several propelinear structures corresponding to the same code $C(H, f)$. For example, the allzero function over $H=\{000,111\}(q=2)$ can be represented as $f\left(x_{1}, x_{2}, x_{3}\right)=0$ or, e.g., as $f\left(x_{1}, x_{2}, x_{3}\right)=x_{1} x_{2}+x_{1} x_{3}$. The resulting code is the same (a 1-perfect Hamming code of length 7); but in the first case, the group is isomorphic to $Z_{2}^{4}$, while the second representation leads to a group isomorphic to $Z_{4} \times Z_{2}^{2}$. The general fact that several propelinear structures can correspond to the same (perfect) code was well demonstrated in [1]. 


\section{B. Transitive Vasil'ev codes of length 15}

There are 201 nonequivalent transitive 1-perfect codes of length 15 [14, Table III]. Five of these codes are Vasil'ev codes, including the linear one; their description can be found in [11] (the four nonlinear codes are denoted by $V 4, V 4^{0}$, $V 4^{1}$, and $V 22^{0} 2^{1}$ ). Let $H$ be spanned by the words $u_{1}=$ $1010101, u_{2}=0111100, u_{4}=0001111, u_{0}=1111111$. Define the functions $f^{V 4}, f^{V 4^{0}}, f^{V 4^{1}}, f^{V 22^{0} 2^{1}}: H \rightarrow\{0,1\}$ by their sets of zeros $\left\{\overline{0}, u_{0}, u_{1}, u_{0}+u_{1}\right\},\left\{\overline{0}, u_{1}, u_{2}, u_{1}+u_{2}\right\}$, $\left\{\overline{0}, u_{0}+u_{1}, u_{0}+u_{2}, u_{1}+u_{2}\right\},\left\{\overline{0}, u_{0}, u_{1}, u_{2}, u_{4}, u_{0}+u_{1}+u_{2}+\right.$ $\left.u_{4}\right\}$, respectively. Then the codes $C\left(H, f^{V 4}\right), C\left(H, f^{V 4^{0}}\right)$, $C\left(H, f^{V 4^{1}}\right), C\left(H, f^{V 22^{0} 2^{1}}\right)$ are representatives of the four equivalence classes of nonlinear transitive Vasil'ev codes of length 15. All these codes are propelinear [1]. Moreover, it can be directly checked that the functions are quadratic:

$$
\begin{aligned}
f^{V 4}(x)= & x_{2} x_{4}+x_{2} x_{6}+x_{4} x_{6}+x_{2}+x_{4}+x_{6}, \\
f^{V 4^{0}}(x)= & x_{1} x_{6}+x_{2} x_{6}+x_{3} x_{6}+x_{1}+x_{2}+x_{3}+x_{6}, \\
f^{V 4^{1}}(x)= & x_{3} x_{5}+x_{3}+x_{5}, \\
f^{V 22^{0} 2^{1}}(x)= & x_{3} x_{4}+x_{3} x_{5}+x_{3} x_{7}+x_{4} x_{5}+x_{4} x_{7}+x_{5} x_{7} \\
& +x_{3}+x_{4}+x_{5}+x_{7} ;
\end{aligned}
$$

so, the corresponding codes meet the hypothesis of Theorem 1 Therefore, all transitive Vasil'ev codes of length 15 belong to the class considered in the current paper.

\section{Transitive functions}

For further development of the topic, it would be interesting to consider a wider class of functions resulting in transitive (propelinear) codes. Such functions should have properties similar to transitivity (propelinearity) of codes:

Problem 1: For a vector space $V$ and a group $\mathcal{A}$ of linear permutations of $V$, find non-quadratic functions $f$ such that for every $c$ from $V$ there exists $\mu \in \mathcal{A}$ meeting $f(\mu(x)+c)=$ $f(x)+l(x)$ for some affine $l$. For instance, for constructing transitive (propelinear) 1-perfect codes as above, we can take $V=H$ and $\mathcal{A} \subset \operatorname{Aut}(H)$.

\section{REFERENCES}

[1] J. Borges, I. Y. Mogilnykh, J. Rifà, and F. I. Solov'eva, "Structural properties of binary propelinear codes," $A d v$. Math. Commun. vol. 6, no. 3, pp. 329-346, 2012, DOI: 10.3934/amc.2012.6.329

[2] J. Borges, I. Y. Mogilnykh, J. Rifà, and F. I. Solov'eva, "On the number of nonequivalent propelinear extended perfect codes," Electr. J. Comb. vol. 20, no. 2, pp. P37 (1-14), 2013. [Online]. Available: http://www.combinatorics.org/ojs/index.php/eljc/article/view/v20i2p37

[3] J. Borges and J. Rifà, "A characterization of 1-perfect additive codes," IEEE Trans. Inf. Theory. vol. 45, no. 5, pp. 1688-1697, 1999, DOI: $10.1109 / 18.771247$

[4] A. E. Brouwer, A. M. Cohen, and A. Neumaier, Distance-Regular Graphs. Berlin: Springer-Verlag, 1989.

[5] C. Carlet, " $Z_{2^{k}}$-Linear codes," IEEE Trans. Inf. Theory vol. 44, no. 4, pp. $1543-1547,1998$, DOI: $10.1109 / 18.681328$

[6] A. R. Hammons, Jr, P. V. Kumar, A. R. Calderbank, N. J. A. Sloane, and P. Solé, "The $Z_{4}$-linearity of Kerdock, Preparata, Goethals, and related codes," IEEE Trans. Inf. Theory vol. 40, no. 2, pp. 301-319, 1994, DOI: $10.1109 / 18.312154$

[7] T. Honold and A. A. Nechaev, "Fully weighted modules and representations of codes," Probl. Inf. Transm. vol. 35, no. 3, pp. 205-223, 1999, translated from Probl. Peredachi Inf., 35(3): 18-39, 1999.

[8] D. S. Krotov, " $Z_{4}$-Linear perfect codes," Diskretn. Anal. Issled. Oper. Ser.1, vol. 7, no. 4, pp. 78-90, 2000, in Russian. Translated at http://arxiv.org/abs/0710.0198

[9] D. S. Krotov, " $Z_{2^{k}}$-Dual binary codes," IEEE Trans. Inf. Theory vol. $53, \quad$ no. 4, pp. 1532-1537, 2007, DOI: 10.1109/TIT.2007.892787

[10] F. J. MacWilliams and N. J. A. Sloane, The Theory of Error-Correcting Codes. Amsterdam, Netherlands: North Holland, 1977.

[11] S. A. Malyugin, "On the equivalence classes of perfect binary codes of length 15," Sobolev Institute of Mathematics, Novosibirsk, Preprint 138, 2004, in Russian.

[12] A. A. Nechaev, "Trace-function in Galois ring and noise-stable codes," in Proc. V All-Union Symp. on Theory of Rings, Alg. and Mod., Novosibirsk, Russia, 1982, p. 97, in Russian.

[13] A. A. Nechaev, "Kerdock code in a cyclic form," Discrete Math. Appl. vol. 1, no. 4, pp. 365-384, 1991, DOI: 10.1515/dma.1991.1.4.365 Translated from Diskretnaya Matematika, 1(4):123-139, 1989.

[14] P. R. J. Östergård, O. Pottonen, and K. T. Phelps, "The perfect binary one-error-correcting codes of length 15: Part II-properties," IEEE Trans. Inf. Theory vol. 56, no. 6, pp. 2571-2582, 2010, DOI: 10.1109/TIT.2010.2046197

[15] V. N. Potapov, "A lower bound for the number of transitive perfect codes," J. Appl. Ind. Math. vol. 1, no. 3, pp. 373379, 2007, DOI: $10.1134 / \mathrm{S} 199047890703012 \mathrm{X}$ translated from Diskretn. Anal. Issled. Oper. Ser. 1, 13(4):49-59, 2006.

[16] J. Rifà, J. M. Basart, and L. Huguet, "On completely regular propelinear codes," in Applied Algebra, Algebraic Algorithms and Error-Correcting Codes, 6th Int. Conference, AAECC-6 Rome, Italy, July 4-8, 1988 Proceedings, ser. Lect. Notes Comput. Sci. Springer-Verlag, 1989, vol. 357, pp. 341-355, DOI: 10.1007/3-540-51083-4 71

[17] J. Schönheim, "On linear and nonlinear single-error-correcting $q$-ary perfect codes," Inf. Control vol. 12, no. 1, pp. 23-26, 1968, DOI: 10.1016/S0019-9958(68)90167-8

[18] F. I. Solov'eva, "On the construction of transitive codes," Probl. Inf. Transm. vol. 41, no. 3, pp. 204-211, 2005, DOI: 10.1007/s11122-005-0025-3 translated from Probl. Peredachi Inf. 41(3):23-31, 2005.

[19] Y. L. Vasil'ev, "On nongroup close-packed codes," in Problemy Kibernetiki, 1962, vol. 8, pp. 337-339, in Russian, English translation in Probleme der Kybernetik, 8: 92-95, 1965. 